\title{
El Libro blanco de la Comisión Europea o el intento de lograr el humanismo tecnológico
}

\section{The White Book of the European Commission or the attempt to achieve technological humanism}

Faustino Gudin Rodríguez-Magariños ${ }^{2}$

Fecha de recepción: 23/05/2020 - Fecha de aceptación: 23/07/2020

DOI : https://doi.org/10.22490/26655489.3819

“El peligro del pasado era que los hombres fueran esclavos. Pero el peligro del futuro es que los hombres se conviertan en robots"

(Erich Fromm).

\section{Resumen}

La IA (Inteligencia Artificial) va trastocando gradualmente toda la vida de la sociedad y, como consecuencia, el derecho que gobierna dicha sociedad. Con el progreso reciente en la adquisición de datos digitalizados, el aprendizaje automático y las infraestructuras informáticas, las aplicaciones de IA se están expandiendo a áreas que anteriormente se consideraban solo una materia para expertos.

Resulta irrelevante, que creamos que la tecnología genera necesidad o la necesidad inventa a la tecnología, el resultado final será idéntico: ya no cabe dar vuelta atrás. La UE está tratando de adaptarse a este nuevo contexto, su objetivo principal, una auténtica tarea hercúlea, se basa en hacer compatibles, por un lado, los derechos fundamentales $\mathrm{y}$, por otro lado, los riesgos que implican la IA. En este artículo, describimos los avances recientes en las tecnologías de IA y sus aplicaciones prácticas, identificamos los desafíos para un mayor progreso

1 Comisión Europea Bruselas, 19 de febrero de 2020 COM (2020) 65 final, actualmente el Libro Blanco está abierto a consulta pública hasta el 19 de mayo de 2020.

2 Pfr. Asociado UAH. Doctor en Derecho. Licenciado en Criminología. Magistrado. Letrado de la Administración de Justicia Excedente. 
en los sistemas de IA y cómo podrían acoplarse en el marco jurídico actual. Por lo tanto, no debemos limitarnos a examinar los aspectos más negativos, pues la tecnología tiene el poder de proteger a todos los ciudadanos europeos (p. ej. controlar las enfermedades transmisibles) $y$, al mismo tiempo, ayuda a consolidar un mercado interior sin fricciones orientado a lograr un mayor nivel de desarrollo conjunto. Sin embargo, detectamos algunos puntos débiles y ciertas lagunas jurídicas en la regulación que tampoco podemos dejar en el tintero.

Palabras clave: IA, Internet de las cosas, edge computing, panopticismo, Derecho e UE

\section{Abstract}

Artificial intelligence ( $\mathrm{AI}$ ) is gradually changing the whole life of society and, as a consequence, the Law who rule this society. With recent progress in digitized data acquisition, machine learning and computing infrastructure, Al applications are expanding into areas that were previously thought to be only a matter of human experts. It is, in addition, irrelevant whether people believe that technology generates need, or need invents technology, the result will be the same: there is no turning back. The EU is trying to adapt to this new scenario, the main goal, a real Herculean task, is based on making compatible, on one hand, respect fundamental rights, and on the other hand, the risks which involve the new techs. In this review article, we outline recent breakthroughs in Al technologies and their practical applications, identify the challenges for further progress in Al systems and how they could couple in the current legal framework. We consider White Paper on Artificial Intelligence a starting point to implement a solid European regulatory framework for trustworthy Al. Therefore, we should not concentrate on the more negative aspects, the technology has the power to protect all European citizens (e. g. controlling communicable diseases) and helps to create a frictionless internal market for further development and, at the same time. Nevertheless, we detect some weak points and legal loopholes that we also cannot fail to mention. and EU.

Keywords: Al, IoT, edge computing, panopticism, Law 


\section{I ntroducción}

Bajo un ángulo negativo, con la irrupción del universo telemático, los peores temores augurados por la célebre novela de George Orwell, 1984, parecen haber cobrado vida en nuestro nuevo escenario vital. La frontera entre el mundo tangible y la ficción entre el mundo virtual y el físico tienden a verse progresivamente difuminadas hasta extremos que nos generan la duda acerca de en qué campo ciertamente nos encontramos ${ }^{3}$. Pero, a su vez, la propia disociación entre la mente humana y la computacional tiende paulatinamente a desvanecerse. En pocos campos como en el relacionado con la IA hallamos que los muros entre el cosmos de la matemática abstracta y su corolario de algoritmos y el mundo de la realidad se tambalean pues, hoy por hoy, se puede afirmar que quien domina los algoritmos puede interferir en todas las esferas de la vida, de tal modo que la vida humana del denominado homo digitalis ya no puede ser comprendido sin ella. Abrazando la tecnología, hemos abierto una caja de pandora de incalculables consecuencias ${ }^{4}$ y al derecho le toca la titánica tarea de instaurar barreras en orden a conservar la individualidad y la dignidad del ser humano.

La IA va a introducir a su vez factores descompensantes en lo que Polibio denominaba "los equilibrios del poder" comprometiendo su irrefrenable expansión, la propia idea de democracia y llegándose a afirmar que nuestras democracias se hallan robotizadas ${ }^{5}$. Así, del mismo modo que la IA puede comprender mejor que los propios humanos los flujos y reflujos

3 En la novela del británico, el M inisterio de la verdad con su doble lenguaje imponía lo que es verdadero y lo que es falso, mediante una estrategia de "hechos alternativos" a una realidad. En este mundo cada vez más globalizado, nos estamos instalando peligrosamente en esa era de la "verdad virtual" en la que los hechos objetivos aparecen desplazados por lo que se dice en la red para los ciudadanos que las apelaciones a las emociones o a las creencias innatas. Las denominadas "fake news" popularizadas por el actual Presidente Donald Trump, vienen a mostrar que existe un manto de desconfianza pues no resulta fácil distinguir entre la realidad y la ficción virtual.

4 Vid. Majó Roca, Joan, Chip, cables y poder: la clase dominante en el siglo XXI, Planeta, Barcelona, 1997, p.36.

5 Cfr. MORENO, Luis/ JIMÉNEZ, Ramón, Democracias Robotizadas. Escenarios futuros en Estados Unidos y la Unión Europea, Eds. Catarata, Madrid, 2018, p. 154. Los autores sostienen que "la robotización de nuestra sociedad no es una mera prospectiva ara nuestras democracias; es algo que está sucediendo y que determina nuestra vida cotidiana. su nivel de propensión se agudizará con el paso del tiempo. Frente a esta aceleración, la democracia se mueve con parsimonia y hasta con torpeza". En definitiva, los autores ponen en duda que estemos suficientemente preparados para gestionar los enormes cambios que la robotización va a comportar en nuestra vida cotidiana. 
del mercado de valores ${ }^{6}$, puede comprender y manipular los estadios de la opinión pública, puede condicionar a la red con bots y crear o manipular ${ }^{7}$ los oportunos estadios de opinión ${ }^{8}$.

Actualmente en la denominada batalla de las inteligencias parece decidida. Ya nadie duda que los ordenadores pueden dar soluciones más apropiadas, e incluso imaginativas, que los humanos a los concretos problemas que se le someten. Baste citar el caso de Deep-Blue contra Kasparov ${ }^{9}$ o de Alpha-Go versus Lee Sedol ${ }^{10}$ para percatarse de que ya no existen cerebros humanos lo suficientemente ${ }^{11}$ preparados para enfrentarse a un ordenador a la hora de resolver dilemas lineales y concretos, comprobándose una tendencia cada vez más desequilibrante para el ordenador. Cabe incluso plantearse que, si cualquier ordenador puede ser mucho más inteligente, imparcial y dar soluciones más ajustadas y creativas que los políticos profesionales, puede que el debate político se limite a elegir un determinado tipo

6 Vid Kamley, Sachin / Jaloree, Shailesh /Thakur, Ramjeevan Singh, "Performance Forecasting of Share Market using Machine Learning Techniques: A Review", International Journal of Electrical and Computer Engineering Vol. 6, Núm. 6, diciembre de 2016, pp. 3.196-3.204.

7 Como apunta Rodrigo Alsina los medios de comunicación pueden crear, sobre todo a partir de la telematización, un clima de opinión de alarma social que, en determinados casos, reforzará la concepción de algunas personas sobre la inseguridad ciudadana". (Vid. Rodrigo Alsina, M iquel, "El conocimiento del sistema penal: alarma social y medios de comunicación", Cuadernos de Derecho Judicial, Núm. IV, 1999, p. 87).

8 Un caso emblemático lo fue el de los bots enviados desde Rusia en favor del Brexit. (Vid. LOMAS, Nastasha, Study: Russian Twiter bots $45 \mathrm{k}$ bots tweets close to vote, 15 de noviembre de 2017, (https://techcrunch.com/2017/11/15/study-russian-twitter-botssent-45k-brexit-tweets-close-to-vote/, última visita 3 de marzo de 2020). MOSTROUS, Alexei/ BRIDGE, Mark/ GIBBONS, Katie, "Russia used Twitter bots and trolls 'to disrupt' Brexit vote", The Sunday Times, 15 de noviembre de 2017, (https://www.thetimes.co.uk/ edition/news/russia-used-web-posts-to-disrupt-brexit-vote-h9nv5zg6c última visita 3 de marzo de 2020).

9 Vid. Krauthammer, Charles, "Be afraid", Washington Examiner, 26 de mayo de 1997, https://www.washingtonexaminer.com/weekly-standard/be-afraid-9802 (última vita 4 de marzo de 2020).

10 Vid. Silver, David / Schrittwieser, Julian/ Simonyan, Karen/ Antonoglou, loannis/ Huang, Aja;/ Guez, Arthur/ Hubert, Thomas/ Baker, Lucas/ LAl, Matthew/ Bolton, Adrian/ Chen, Yutian/ Lillicrap, Timothy;/ Fan, Hui/ Sifre, Laurent/ Driessche, George van den/ Graepel, Thore/ Hassabis, Demis, "Mastering the game of Go without human knowledge", Nature, Vol. 550, Núm. 7676, 19 de octubre de 2017, pp. 354-359.

11 Vid. Fatemeh, Zahedi, "An Introduction to Neural Networks and a Comparison with Artificial Intelligence and Expert Systems", Interfaces, Vol. 21, Núm. 2, marzo/abril, 1991, pp. 25-38. 
de ordenador. La deshumanización de la vida cotidiana es una parte del proceso tecnológico, no podemos solo acogernos a sus ventajas y renunciar a sus inconvenientes.

Pero, más allá del debate sobre la manipulación del poder al sometido, Orwell, recoge en su novela la arcaica idea egipcia de Horus "el ojo que todo lo ve", que se pasa a ser el Gran Hermano que "todo lo ve", auspicia la potencial llegada de un opresivo sistema donde cualquier mínimo gesto, movimiento o pensamiento del individuo pasa a estar controlado ${ }^{12}$. Por eso en la teología judeocristiana existe una tautología o pleonasmo: “Dios es omnisciente porque es omnipotente"13. Poder y conocimiento son dos caras de la misma moneda, realmente Foucault ${ }^{14}$ afirma que la relación entre conocimiento y poder es simplemente lingüística y no existe una diferencia real.

A modo de aproximación la IA (Artificial Intelligence, - Al) puede ser reputada como la simulación de procesos de inteligencia humana efectuada por parte de máquinas, especialmente sistemas cibernéticos que se proyectan a los sistemas telemáticos. Estos procesos incluyen el aprendizaje (la adquisición de información y reglas para el uso de la información), el razonamiento (usando las reglas para llegar a conclusiones aproximadas o definitivas) y la autocorrección. Las aplicaciones particulares de la Al incluyen sistemas expertos, reconocimiento de voz, así como la visión artificial.

Todos los aspectos de la vida, cual rostro de Jano, suelen tener dos caras, tal como el Libro Blanco indica, la IA implica una serie de riesgos potenciales, como la toma de decisiones poco transparentes, diversos tipos de discriminación, intrusión en nuestra vida privada o ser utilizado con fines criminales.

12 Vid. Wilson, Hilary, Understanding Hieroglyphs: A Complete Introductory Guide, Michael O’ Mara Books Ltd,. Londres, 1965, p. 165.

13 "Los ojos de Jehová están en todo lugar, mirando a los malos y a los buenos" (Libro de los Proverbios 15: 3).

14 Vid. Foucault, Michel, Microfísica del poder, Ediciones de La Piqueta, Madrid, 1979, pp. 177. El planteamiento de Foucault es tomado de Francis Bacon quien es el primero en defender la equiparación real entre ambos términos. 
Para Weizenbaum ${ }^{15}$ ninguna máquina debería nunca tomar decisiones de forma completamente autónoma o dar consejos que requieran, entre otras cosas, de la sabiduría, producto de experiencias humanas, así como de tener en cuenta valores humanos. La tecnología y la vida digital comportan insoslayables desafíos éticos y jurídicos ${ }^{16}$.

\section{I nteligencia e I A}

Como refiere Clutterbuck ${ }^{17}$ en inglés la palabra "intelligence" (inteligencia) comporta dos acepciones con dos significados aparentemente diversos: información (sobre adversarios o sobre sucesos) y la equivalente al castellano vulgar capacidad de comprender. Sin embargo, estos dos términos aparentemente antitéticos convergen de una forma realmente sorprendente cuando hablamos de IA. Por ejemplo, los chips neuromórficos toman por modelo estructuras biológicas como el cerebro donde el cerebro sigue unas reglas internas de codificación donde el estímulo físico viene seguido de fenómenos que transforman el estímulo físico en impulsos nerviosos generando una respuesta a esta señal en forma de percepción o experiencia consciente a esa sensación ${ }^{18}$. La complejidad del cerebro humano es tal que ni siquiera puede decirse que el cerebro de un individuo sea cualitativamente igual durante toda su vida. Modernamente se empieza a ver que el cerebro va evolucionando con el paso de los años que es un órgano plástico que se va modulando y reacondicionado

15 Vid. Weizenbaum, Joseph, Computer Power and Human Reasoning: From Judgment to Calculation, San Francisco, W. H. Freeman and Company, 1976, pp.42 y ss.

16 A modo de botón de muestra, cabe citar el descubrimiento del «uso sin consentimiento de la información» que involucró a la consultora Cambridge Analytica, consultora británica cuyo objetivo es moldear el comportamiento de su audiencia a través de la minería, canalización y uso de datos. En 2016 accedió a la información de alrededor de 50 millones de usuarios de Facebook, dichos datos se habrían utilizado para beneficiar a Donald Trump en su campaña presidencial, creando una polémica que puso en duda la relación de los usuarios y su información con internet. (Vid "Cambridge Analytica and Facebook: The Scandal and the Fallout So Far", New York Tomes, 4 de abril de 2018. https://www.nytimes.com/2018/04/04/us/politics/cambridge-analytica-scandal-fallout.html).

17 Vid. Clutterbuck, Richard, Terrorism in an unstable world, Londres/ Nueva York, 1994, p. 64.

18 Vid. Gardner, Esther P., / Martin, John H., "Codificacion de la informacion sensorial" en Principios de neurociencia (coords. Kandel, Erik R et all), Mc Graw Hill, 1a Ed., Madrid, 2001, pp. 411-429. 
continuamente. Tanto es así que autores como Mora ${ }^{19}$ constatan un desarrollo psíquico intelectual que hace que en cada fase de la vida un mismo individuo pueda ser considerado cualitativamente distinto, la historia evolutiva por su parte ha ido facilitando la creación de determinados atributos ${ }^{20}$.

Para Pinker ${ }^{21}$, el funcionamiento del cerebro y el funcionamiento IA son dos mundos disociados. Pero habría que consensuar un término unívoco para la IA. En primer lugar, cabría distinguir entre una IA fuerte y débil. Quien introdujo esta distinción entre la IA débil y fuerte fue el filósofo John Searle en un artículo crítico sobre la IA publicado en $1980^{22}$ que estimuló, y sigue excitando, un enconado debate. La IA fuerte implicaría que un ordenador convenientemente diseñado no simula una mente, sino que actúa como una mente y por consiguiente debería ser capaz de tener una inteligencia igual o incluso superior a la humana; este concepto es al que vamos a restringirnos.

La UE ${ }^{23}$ ha dado cuerpo al término «IA» reputándolo como un sistema que manifiesta un comportamiento inteligente, pues son capaces de analizar su entorno y pasar a la acción - con cierto grado de autonomía- con el fin de alcanzar objetivos específicos.

Todos los avances de la investigación en IA se han centrado en construir inteligencias artificiales especializadas y los éxitos alcanzados son muy impresionantes, en particular durante el último decenio gracias sobre todo a la conjunción de dos elementos: la disponibilidad de enormes cantidades de datos y el acceso a la computación de altas prestaciones para poder analizarlos.

19 Vid. Mora, Francisco, El reloj de la sabiduría, Tiempos y espacios en el cerebro humano, Alianza Ed., Madrid, 2002, pp. 65-66.

20 Así en palabras de Pinker: "nuestra mente, nuestro cerebro, equipado por la adaptación evolutiva para sobrevivir en un mundo prehistórico, se enfrenta a las paradojas de la ciencia y la vida moderna al no haber sido dotado con herramientas para comprenderlas intuitivamente". [Vid. PINKER, Steven, La tabla rasa: La negación de la naturaleza humana, (trad. Filella Escolà, Roc), Paidós, Barcelona, 2003, cap. 13].

21 Cfr. Pinker, Steven, Cómo funciona la mente, (trad. Ferran Meler-Orti), Destino, Barcelona, 2008, pp. 39-40.

22 Vid. Searle, John R., "M inds, brains, and programs", Behavioral and Brain Science, Vol. 3, Núm.3, 1980, pp. 417-457.

23 Bruselas, 25 de abril de $2018 \operatorname{COM}(2018) 237$ final, p.1. 
Efectivamente, el éxito de sistemas, como por ejemplo AlphaGo ${ }^{24}$ o Watson ${ }^{25}$ y los avances en vehículos autónomos o en los sistemas expertos de diagnóstico médico basado en imágenes, han sido posibles gracias a esta capacidad para analizar grandes cantidades de datos y detectar patrones eficientemente. Sin embargo, prácticamente se considera ${ }^{26}$ que no hemos avanzado hacia la consecución de la IA completa. En realidad, podemos aseverar que los actuales sistemas de IA son una demostración de lo que Daniel Dennet Ilama "competencia sin comprensión"27.

De otro lado, comparando la IA con el cerebro humano Saxena ${ }^{28}$ razona que los ordenadores conllevan un gasto masivo de energía. El autor propugna crear nuevos algoritmos de aprendizaje que no requieran enormes cantidades de datos para ser entrenados, así como un hardware mucho más eficiente en consumo energético para implementarlos, ya que el consumo de energía podría acabar siendo una de las principales barreras al desarrollo de la IA. En comparación, el cerebro es varios órdenes de magnitud más eficiente que el hardware actual necesario para implementar los algoritmos de IA más sofisticados. Una posible vía por explorar es la computación neuromórfica basada en memristores ${ }^{29}$.

24 Vid. Silver, David/ Huang, Aja/ Maddison, Cristopher J../ Guez, Arthur/ Sifre, Laurent/ Ven Den Driessche, George/ Schrittwieser, Julian/ Antonoglou, Ioannis/ Panneershelvam, Veda/ Lanctot, Marc/ Dieleman, Sander/Grewe, Dominik/ Nham, John / Kalchbrenner, Nal / Sutskever, Ilya/ Lillicrap, Timothy/ Leach, Maddy / Kavukcuoglu, Koray/ Graepel, Thore / Hassabis, Demis, "Mastering the game of go with deep neural networks and tree search", Nature, Vol. 529, Núm. 7.587, 2016, pp. 484-489.

25 Vid. Ferrucci, David A. / Levas, Anthony/ Bagchi, Sugato/ Gondek, David / Mueller, Erik T. “Watson: beyond jeopardy!", Artificial Intelligence, Núm. 199, 2013, pp. 93-105.

26 Vid. Lake, Brenden M / Ullman, Tomer D./ Tenenbaum, Joshua B/ Gershman./ Samuel J., "Building machines that learn and think like people", Behavioral and Brain Sciences, Vol. 40, 24 de noviembre de 2016.

27 Vid. Dennet, Daniel C., From Bacteria to Bach and Back: The Evolution of Minds, Penguin Random House Londres, 2018.

28 Vid. Saxena, Visal/ Wu, Xinyu/ Srivastava, I ra / Zhu, Kehan, “Towards neuromorphic learning machines using emerging memory devices with brain-like energy efficiency, preprints", Preprint v2. 6 de septiembre de 2018.

29 Vid. https://revistapesquisa.fapesp.br/es/2017/10/26/el-secreto-de-los-mem ristores/ (última visita 8 de marzo de 2020). 


\section{Los peligros de la I A para los derechos fundamentales: El caso del crédito social chino}

Cabe afirmar que los ciudadanos digitales chinos viven en un cosmos particular digital donde todo lo que pueden ver, producir o compartir, está limitado por legislaciones, filtros, rastreo, censores humanos todo ello analizado por un sistema oficial de IA, que da sentido a los innumerables datos recopilados de sus ciudadanos tanto dentro de la red como fuera de ella. El Sistema de Crédito Social, fue instaurado el 14 de junio de 2014 cuyo fin es lograr la «armonía social» 30 , según el documento oficial del Consejo de Estado. Su propósito oficial consiste en "establecer mecanismos de incentivo para la autocorrección y la automejora que se enfoquen en disminuir los actos que quiebren la confianza, y promover mecanismos de investigación de infracciones al sistema de crédito, además de castigar severamente la filtración al extranjero de secretos financieros o estatales orientado a fortalecer el rol de la supervisión social"31.

El gigante asiático se ha convertido en un gigante telemático que, además, al no hallarse encorsetado por el respeto a ninguna garantía democrática, despliega todas las potencialidades de vigilancia de sus sistemas sobre su ciudadanía a la que paulatinamente expone a un férreo y draconiano grado de control ${ }^{32}$. Así China está aglomerando una infraestructura de supervigilancia cibernética con un sistema de puntuación similar al que se usa comúnmente para la evaluación financiera, análogas a instituciones tales como Equifax, o para la evaluación de riesgo de clientes bancarios.

30 Vid. “Use Big Data Thinking and Methods to Enhance the Government's Governing Capacity", China Copyright and Media, 2019, https: //chinacopyrightandmedia.wordpress. com/2016/07/12/use-big-data-thinking-and-methods-to-enhance-the-governmentsgoverning-capacity/(última vista el 6 de marzo de 2020).

31 Vid. "Planning Outline for the Construction of a Social Credit System (20142020)", China Copyright and Media, 2014, https://chinacopyrightandmedia. wordpress.com/2014/06/14/planning-outline-for-the-construction- of-a-social-creditsystem-2014-2020/. (última vista el 6 de marzo de 2020).

32 Se ha popularizado la analogía entre el crédito social chino y el famoso primer episodio de la tercera temporada de Black Mirror de la serie de Netflix "Nosedive". (Sobre este tema vid. Business Insider, China might use data to create a score for each citizen based on how trustworthy they are, recopilado el 21 de febrero de 2019, disponible en https: // www. businessinsider. com/china-social-credit-score-like-black-mirror-2016-10, (última visita el 6 de marzo de 2020). 
La vigilancia y los métodos de «premio y castigo» no se basan solo en el cumplimiento de unas expectativas financieras, sino que son parte de una evaluación moral y su propósito tiene consecuencias peligrosas desde el plano del respeto a la individualidad y a la vida privada. De este modo, sustentado en una larga tradición «confuciana» de control social, el Gobierno está constantemente supervigilando y evaluando a los ciudadanos de modo que los categoriza dependiendo del modo en que se acoplan a las expectativas oficiales. De esta manera sabe si los ciudadanos son «de confianza», creen en las ideas del partido y son consecuentes con estas. No en vano cabe recordar el lema oficial: «el Gobierno pone el escenario, el pueblo canta en armonía»33.

Tras su irrupción como programa piloto en 2015, el Crédito Sésamo ha sido presentado como una especie de juego comunitario (gamification). Los usuarios compiten por ser los mejores ciudadanos y cada individuo tiene una cuenta que asocia un puntaje a cada comentario, compra, palabra buscada o app descargada ${ }^{34}$. En definitiva, la IA analiza todos los datos recopilados derivados del comportamiento online (e incluso offline), en todas las plataformas ${ }^{35}$. En consecuencia, cada usuario arroja una puntuación que se va acumulando, alcanzar una buena puntuación implica lograr la etiqueta de buen ciudadano lo que comporta importantes consecuencias prácticas, el mal desempeño en este «juego» puede ser etiquetado dentro de las «listas negras» de deuda comportamiento ${ }^{36}$.

33 Vid. "Use Big Data Thinking and Methods to Enhance the Government's Governing Capacity", China Copyright Media 2016, disponible en https://chinacopyrightandmedia. wordpress.com/2016/07/12/use-big-data-thinking-and-methods-to-enhance-thegovernments-governing-capacity//. (última vista el 6 de marzo de 2020).

34 Vid. McDonald, Joe, "China bars millions from travel for 'social credit' offenses", Associated Press, 23 de marzo de 2019, (vid. https://apnews.com/9d43f4b74260411797 043ddd391c13d8). (última visita el seis de marzo de 2020).

35 Un caso emblemático lo encontramos con la aplicación WeChat, que en el país asiático posee más de mil millones de usuarios. Además, se han creado empresas dedicadas al procesamiento de datos para afianzar el sistema de crédito socialVi, XinhuaNet News, "China's first private credit-scoring firm starts operation", 23 de mayo de 2018, (vid. http://www.xinhuanet.com/english/2018-05/23/c_137201204.htm, última visita el seis de marzo de 2020).

36 XinhuaNet News, "China Focus: Chinese courts use technology to tighten noose on debt defaulters", 2017, vid. http://www.xinhuanet.com/english/201710/03/c_136657135.htm(última visita el seis de marzo de2020). 


\section{Datos básicos para comprender el documento: EI I OT y la edge computing}

Dos conceptos asociados a la IA son la IOT y la edge computing. El Ilamado IoT $^{37}$ ("internet de las cosas"), concepto que alude a la interconexión de toda clase de aparatos domésticos con internet. Los referidos aparatos continuamente van suministrando un volumen impresionante de datos sobre los usuarios, en el mejor de los casos, podrán ser empleados con fines torticeros. En virtud de que el acopio y análisis de la masiva cantidad de información generada a través de la interacción de los diversos dispositivos y objetos, tiene un alto valor comercial dentro de este esquema de hiperconectividad, lo que hace atractiva su obtención ilícita ${ }^{38}$.

No obstante, estas aplicaciones no siempre van en contra de los intereses de los individuos, sino que el denominado internet de las cosas puede permitir que se evite cuidar de nuestros ancianos, denunciar y prevenir accidentes domésticos y garantizar peligros para nuestra salud ${ }^{39}$. Ningún país puede renunciar a incorporar los adelantos de la IA, porque su rechazo revertiría en una profundización de "la brecha tecnológica" 40 , con una menor competitividad, por lo tanto el problema aparece mayormente enfocado más que en su aceptación o no, en su encuadre en los regímenes democráticos, garantistas y respetuosos, de los derechos fundamentales.

Es de todos conocido que el Big Data sobredimensiona su poder con billones de datos anodinos obtenidos sinuosamente que pueden aportar cantidades masivas de información bruta. Los programas proporcionan masivos datos a la I A cuyo resultado

37 Acrónimo del término inglés "internet of things". El concepto de internet de las cosas lo propuso Kevin Ashton en el Auto-ID Center del MIT en 1999. http://www. rfidjournal.com/articles/view?4986

38 Así los primeros pasos del denominado internet de las cosas se sitúan en el año 1990 cuando John Romkey y Simon Hacket consiguieron diseñar una tostadora con conectividad a Internet, pudiendo desde cualquier ordenador determinar su encendido, su apagado y configurar el tiempo de tostado de esta.

39 Sobre este tema vid. Islam, S. M. Riazul/ Kwak, Daehan; Kabir MD. Humaun/ Mahmud, Hossain / Kyung-Sup, Kwak, "The Internet of Things for Health Care: A Comprehensive Survey", IEEE Access, Vol. 3, 1 de junio de 2015, pp. 678-708.

40 Vid. Hilber, Martin, R., "From industrial economics to digital economics». CEPAL. United Nations Publicatio", , Ponencia del XIII Congreso de Derecho e Informática FIADI, Lima, noviembre 2009. 
final resulta una cosificación donde se tiende a difuminar la distinción entre persona y el ordenador, entre sujeto y objeto comprometiendo indirectamente valores como la dignidad humana consagrada en el art.10 CE.

Un paso más allá es el denominado (edge computing) que busca maximizar la información del IoT. Los millones de dispositivos del I oT que nos rodea tienen un problema: recolectan información, pero no hacen nada con ella. La envían a la nube, donde grandes centros de datos la procesan para obtener ciertas conclusiones o activar ciertos eventos.

Ese funcionamiento "pasivo" de los sistemas de IA es lo que quiere cambiar la llamada Edge Computing, un tipo de filosofía aplicable especialmente en escenarios empresariales e industriales que aporta mucha más autonomía a todos esos dispositivos, haciendo que sean analíticamente activos. Hasta ahora, las grandes plataformas de Cloud Computing se encargaban de hacer es especie de "trabajo sucio" de analizar los datos recolectados por los sensores y dispositivos IOT. EI sistema permite que los datos producidos por los dispositivos del I oT se procesen por la IA más cercana a donde se crearon en lugar de enviarlos a través de largos recorridos para que lleguen a centros de datos y nubes de computación, lo que permite a las organizaciones analizar los datos importantes casi en tiempo real, algo que deviene a ser una auténtica necesidad en muchos sectores estratégicos tales como la fabricación, la salud, las telecomunicaciones o la industria financiera.

Firmas como McKinsey \& Co. ${ }^{41}$ estiman que la llamada Industrial Internet of Things (IOT) producirá ingresos de 7,5 billones de dólares en 2025. Otro concepto asociado es la llamada Fog Computing. Como revelaba un estudio de $\mathrm{Cisco}^{42}$, esta plataforma permite "extender la nube para que esté más cerca de las cosas que producen y se accionan mediante datos de dispositivos IoT".

41 Vid. https://www.xataka.com/internet-of-things/edge-computing-que-es-y-porque-hay-gente-que-piensa-que-es-el-futuro (última visita el 6 de marzo de 2020).

42 Vid. https://www.cisco.com/c/dam/en_us/solutions/trends/iot/docs/ computing-overview.pdf (última visita el 6 de marzo de 2020 ). 
Por su parte el propio Libro Blanco aporta importantes datos en este sentido. El texto del documento afirma que el volumen de datos producidos en el mundo está creciendo rápidamente, de 33 zettabytes en 2018 a 175 zettabytes esperados en $2025^{43}$. El documento analizado entiende que cada nueva ola de datos trae oportunidades para que Europa se posicione en la economía de datos ágiles y se convierta en un líder mundial en esta área; el $80 \%$ del procesamiento y análisis de datos que tiene lugar en la nube ocurre en centros de datos e instalaciones informáticas centralizadas, y $20 \%$ en objetos inteligentes conectados, como automóviles, electrodomésticos o robots de fabricación, y en instalaciones informáticas cercanas al usuario ("edge computing"). Según el Libro Blanco, para 2025 estas proporciones cambiarán notablemente ${ }^{44}$.

Se afirma que Europa es el líder mundial en electrónica de baja potencia lo que resulta clave para la próxima generación de especialistas procesadores para IA. Este mercado se encuentra dominado actualmente por operadores no pertenecientes a la UE. Esto podría cambiar con el ayuda de iniciativas como la European Processor I nitiative, que se centra en el desarrollo de baja potencia, sistemas cibernéticos para informática de alto rendimiento de última generación y el trabajo de la empresa común Key Digital Technology, propuesta para comenzar en 2021. Europa también lidera soluciones neuromórficas ${ }^{45}$ que son ideales para automatizar procesos industriales (así la industria 4.0) y modos de transporte. Como resultado se entiende que Europa pueden mejorar la eficiencia energética en varios órdenes de magnitud.

$43 \operatorname{COM}(2018) 795$.

$44 \mathrm{Vid}$. https://info.claroty.com/claroty-gartner-ot-security-best-practices?utm medium $=p p c \& u t m$ term=gartner\&utm_campaign=Gartner+OT+Security + Best $+\bar{P}$ ractices\&utm_source=adwords\&hsa_k $\bar{w}=$ gartner\&hsa_cam $=8121311945 \& \mathrm{hsa}$ ver=3\&hsa_acc=9787177094\&hsa_ad $=\overline{3} 97637323197 \&$ hsa_grp $=92331794348 \&$ hsa_ src $=g \& h s a+m t=b \& h s a+t g t=k w d-18045479368 \& h s a \_n e t=a d w o r d s \& g c l i d=C j O K C Q i A 1-$ 3yBRCmA RIsAN7B 4 H 10 jekG__P_C8IKCw9 4 v7tpniw 8 HoynXUCXeqpUOwpdgOWnRneB5gaAkNOEALw_wc $\bar{B}$ (última visita 12 de marzo de 2020).

45 Las soluciones neuromórficas es un concepto que implica que cualquier sistema de circuitos integrados a gran escala tiende a imitar arquitecturas neurobiológicas presente en el sistema nervioso. 


\section{Primeros pasos de la UE}

La IA ha figurado en los programas marco de investigación y desarrollo de la UE desde 2004, prestándose especial atención a la robótica. Las inversiones se incrementaron hasta los 700 millones EUR para el período 2014-2020, y se complementaron con 2100 millones EUR de inversiones privadas como parte de una asociación público-privada en materia de robótica ${ }^{46}$. Estos esfuerzos han contribuido significativamente a la posición de liderazgo en el campo público de Europa en el ámbito de la robótica.

Hace unos tres años se inició en el ámbito de la UE un manifiesto en relación con una posible normativa que aborde diversas cuestiones relacionadas con los robots y la $I A^{47}$. La referida iniciativa ha tenido un evidente impacto en los medios de comunicación; si bien dicha atención se ha visto fundamentalmente focalizada en los asuntos en los que más se ha hecho mayor empeño, sean los aspectos relacionados con el tema que más directamente atañe a los ciudadanos como son los recortes en el empleo, y muy especialmente la potencial correlación entre la pérdida de puestos de trabajo por la incorporación de robots en detrimento del capital humano e incluso en la posibilidad de que esos nuevos robots coticen de alguna manera ante la seguridad social ${ }^{48}$.

El alto interés del Parlamento de la Unión Europea por abordar estas cuestiones ${ }^{49}$ ha comportado inclusive una iniciativa

46 Vid. https://www.eu-robotics.net/sparc/ (última visita 6 de marzo de 2020)

47 Vid. «Robots e IA: eurodiputados piden normativa comunitaria», Nota de prensa del Parlamento Europeo del 09/02/2017 disponible en: http://www.europarl. europa.eu/news/es/news-room/plenary/2017-02-13/8; (última consulta en web el 14 de febrero de 2017). El Proyecto de Informe, con recomendaciones destinadas a la Comisión sobre normas de Derecho civil sobre robótica, de 31 de mayo de 2016, de la Comisión de Asuntos Jurídicos del Parlamento Europeo, puede consultarse desde: http://www.europarl.europa.eu/sides/getDoc.do?pubRef=-\%2F\%2FEP\%2F\%2FNONSG ML\%2BCOMPARL\%2BPE-582.443\%2B01\%2BDOC\%2BPDF\%2BV0\%2F\%2FES.

48 Vid. Fernández Hernández, Carlos B, «El Parlamento Europeo presenta unas recomendaciones sobre normas de derecho civil sobre robótica», Noticias Jurídicas del 24/11/2016 disponible en: http://noticias.juridicas.com/actualidad/noticias/11464-elparlamento-europeo-presenta-unas-recomendaciones-sobre-normas-de-derecho-civilsobre-robotica/; (últimas consultas de todos loen enlaces url, el 4 de marzo de 2020).

$49 \quad$ Vid.

http://www.europarl.europa.eu/news/es/news-room/ plenary/2017-02-13/8; (última consulta en web, el 4 de marzo de 2020). 
de consulta popular ${ }^{50}$ a los ciudadanos sobre la regulación de los robots y la IA, encuesta on-line a la que se puede acceder del 8 de febrero al 30 de abril de $2017^{51}$. El 8 de abril de 2019, el grupo de expertos de alto nivel en IA presentó Pautas Éticas para una IA Confiable. A ello le siguió a la publicación del primer borrador de las directrices en diciembre de 2018 en el que se recibieron más de quinientos comentarios a través de una consulta abierta.

Tras ello el 8 de abril de 2019 la sección estratégica de la comisión publicó su informe definitivo sobre "Pautas éticas para una IA confiable" ${ }^{\prime 52}$. El documento se basa en ofrecer una serie de directrices que configuran un bloque de siete exigencias claves para que los sistemas de IA puedan ser considerados confiables. Una lista de evaluación específica que comporta como objetivo ayudar a verificar la aplicación de cada uno de los siete requisitos clave: actuación y supervisión humana, robustez técnica y seguridad, privacidad y gestión de los datos, transparencia, diversidad, no discriminación y equidad, bienestar social y medioambiental y responsabilidad.

Anteriormente cabe citar el Plan de Acción E-justicia 20092013 53 prorrogado por el Proyecto de Estrategia 2014-2018 relativa a la Justicia en Red Europea ${ }^{54}$, el cual no dejó de ahondar en la parte más técnica del Plan de Acción pero que repite sus mismos objetivos estratégicos, y que supone una clara línea de continuidad respecto al mismo. Un proyecto ambicioso que pretende otorgar esa libre circulación a la justicia por lo largo y ancho del territorio europeo, gracias a la mejora del acceso a la

50 Vid. Meler Gines, Nuria/Fernández Hernández, Carlos B.. «El Parlamento Europeo consulta a los ciudadanos sobre la regulación de los robots y la IA», Diario $L A L E Y$, Ed. Wolters Kluwer, 09 de febrero de 2017 disponible en http://diariolaley. laley.es/Content/Documento.aspx?params=H4sIAAAAAAAEAGWMsQqDMBRFv8YsLkk slQ6ZtEOHIg7u5SXvhT4qiUQj-PeNS0G6XC73Hs4CdjZaVq3Tag8twC0Zxj46o_bOKw1gC yIYOTyuUkql1bIRWqyUZo6hfKqVWI5EiEhD35kckDwHwh1hvXXZsE1kPIwzCbIxfg6m18 gJjZ3CvmZODieYDxVDRyHwuA_c0s11B1bSkiJ3Dt-AfDHQR7ZAAAAWKE (última consultā en web, el 14 de marzo de 2020)

51 Vid. https://opinio.secure.europarl.europa.eu/opinio/s?s=secured_robotics; (última consulta en web, el 4 de marzo de 2020).

52 Vid. https://ec.europa.eu/digital-single-market/en/news/ethics-guidelinestrustworthy-ai (última visita el 8 de marzo de 2020).

53 Vid. D.O.U.E 31 de marzo de 2009. IV. Plan de Acción Plurianual 2009-2013 relativo a la justicia en la red Europea [DOUE C 75/01, 31.3.2009].

54 Vid. DOCE de 21 de diciembre de 2013. 
justicia y a fomentar la cooperación entre autoridades judiciales de toda la UE a través de herramientas concretas entre las que destaca la posibilidad de la mediación on line.

Se empiezan a acuñar términos tales como la llamada «Cuarta Revolución Industrial». El informe del Forum Económico Mundial, de enero de 2016, sobre «The Future of Jobs: Employment, Skills and Workforce Strtategy for the Fourth Industrial Revolution», matizaba datos interesantes sobre esta cuestión ${ }^{55}$.

Así, se marca una tendencia de un incremento del $9 \%$ en avances en robótica y transportes autónomos, para el marco de tiempo referencial de los años 2018 al 2020. Pero esta cuestión que ahora, en el siglo XXI, parece novedosa, no lo es tanto si miramos a la década finales de los 80 del siglo pasado, cuando ya algún autor vaticinaba estos importantes cambios $^{56}$, y eso teniendo en cuenta que tanto el desarrollo de la robótica como de la IA, no estaba tan avanzado como en la actualidad.

Otro enfoque importante en el ámbito de la UE lo encontramos en la estratégica área de la protección de datos. Cuando el interesado se ve sometido a decisiones que afectan jurídicamente a cualquiera de los aspectos de su vida y estas decisiones se toman «únicamente» por el tratamiento de datos automatizado de datos personales, entra en juego la aplicación del art. 22 del RGPD. En estos casos, las decisiones automatizadas son tomadas por aplicaciones basadas en IA que aprenden de las interacciones con las personas ( $y$, sobre todo, de sus datos personales), para así alcanzar el fin para el que fueron diseñados. Conforme al artículo señalado, el interesado tiene derecho a no verse sometido a una decisión tomada exclusivamente por una máquina guiada por IA. Para que el consentimiento pueda ser considerado válido este debe, por lo menos, respetar las características de ser libre, específico, informado e inequívoco, tal y como se detalla en la definición prevista en el art. 4.11 RGPD.

De otro lado, la I A toca el acquis o acervo comunitario desde muy variados frentes. así forma parte de la Estrategia de la

55 Vid. http://www3.weforum.org/docs/WEF_Future_of_Jobs.pdf (en inglés) (Fecha de última consulta en web, el 14 de febrero de 20̄17).

56 Vid. Lasierra Esteban, José M anuel, «El fin del fin del trabajo», Revista de Acciones e Investigaciones Sociales, Núm 6, 1997, pp. 67-82. 
Comisión para la digitalización de la industria ${ }^{57}$ y de la Estrategia renovada de política industrial de la $U^{58}$. El documento advierte que Europa se sitúa a la zaga en inversiones privadas en IA, las cuales oscilaron entre 2400 y 3200 millones de euros en 2016, frente a 6500 - 9700 millones de euros en Asia y 12100 - 18 600 millones de euros en América del Norte ${ }^{59}$.

\section{La estrategia europea para la I A presentada en abril de $2018^{60}$}

Este documento parte de afirmar las bondades y beneficios de la IA, así como que no hay posibilidad de dar marcha atrás en un mundo cada vez más tecnológico y digitalizado. De otro lado recuerda que estos avances se tienen que adaptar a los principios y valores propios de la UE y no al revés ${ }^{61}$.

El documento concluye que la UE parte de una sólida base industrial y científica, derivada de las avanzadas universidades, así como con laboratorios de investigación punteros, con un reconocido liderazgo en robótica, así como con empresas emergentes innovadoras. Dispone de un marco jurídico completo, que protege a los consumidores al tiempo que favorece la innovación, y está avanzando en la creación de un mercado único digital. Reúne los principales ingredientes para convertirse en líder de la revolución de la IA, de acuerdo con su propia visión y basándose en sus propios valores.

$57 \operatorname{COM}(2016) 180$ final.

$58 \operatorname{COM}(2017) 479$ final.

59 El documento se apoya en el informe aportado por James Mayinka para el McKinsey Global Institute, titulado "10 imperatives for Europe in the age of Al and automation, ", de octubre de 2017 (vid. https://www.mckinsey.com/featured-insights/ europe/ten-imperatives-for-europe-in-the-age-of-ai-and-automation, última vista 4 de marzo de 2020.

60 Bruselas, 25 de abril de $2018 \operatorname{COM}(2018) 237$ final.

61 Así el Artículo 2 del Tratado de la UE: «La Unión se fundamenta en los valores de respeto de la dignidad humana, libertad, democracia, igualdad, Estado de Derecho y respeto de los derechos humanos, incluidos los derechos de las personas pertenecientes a minorías». Los Estados miembros tienen en común una «sociedad caracterizada por el pluralișmo, la no discriminación, la tolerancia, la justicia, la solidaridad y la igualdad entre mujeres y hombres». 
El texto realza la necesidad de aunar fuerzas a nivel europeo y generar sinergias, a fin de garantizar que todos los europeos formen parte de la transformación digital, que se dediquen recursos suficientes a la IA y que los valores y los derechos fundamentales de la Unión figuren en la vanguardia de las actividades relacionadas con ella. Entre todos, podemos conseguir poner al servicio del progreso humano el potencial de la IA.

\section{La estrategia europea para los datos de $2020^{62}$}

Publicada con la misma fecha que el Libro Blanco, debe ser interrelacionada con él. Busca convertir la UE en líder de una sociedad impulsada por los datos. La creación de un mercado único de datos que viabilice que estos fluyan libremente por la UE y entre sectores, en beneficio de las empresas, los investigadores y las administraciones públicas. En consecuencia, la a UE propugna la creación de un mercado único de datos donde:

a. Los datos puedan circular por toda la UE y entre sectores, en beneficio de todos.

b. Se respeten plenamente las normas europeas, en particular en materia de privacidad y protección de datos, así como la legislación sobre competencia.

c. Las normas para el acceso a los datos y su utilización sean justas, prácticas y claras.

Se prevé para 2025 un incremento del volumen global de datos en un 530\% (pasando de 33 zetabytes en 2018 a 175 zetabytes). Un incremento a 829000 millones de euros en que se cifra el valor de la economía de los datos en la EU, frente a 301000 millones de euros (2,4\% del PIB de la UE) en 2018. Un ascenso en el mercado laboral que va de 10,9 millones de profesionales de los datos en la EU frente a 5,7 millones que había en 2018 . Finalmente, se calcula en un $65 \%$, el porcentaje de la población de la UE con competencias digitales básicas frente al 57\% que existía en 2018. 
La estrategia europea de datos de la Comisión describe las medidas de política y las inversiones necesarias para que la UE compita en el mercado global y digital al tiempo que conserva sus altos estándares de privacidad y seguridad. La comunicación señala la fragmentación y los desequilibrios del mercado como posibles obstáculos. Sin embargo, también establece cómo pretende crear un "verdadero espacio de datos europeo" mediante el establecimiento de un marco regulatorio para la gobernanza, el acceso y la reutilización de datos entre empresas, entre empresas y gobiernos, y dentro de las administraciones.

\section{El Libro Blanco para la I A de 2020}

Este documento persigue, desde un enfoque muy europeo, convertir a la Unión Europea en líder global de la data-economy y sus aplicaciones, en beneficio de ciudadanos, negocios y sociedad en general, sobre la base de dos pilares fundamentales: de un lado promover la IA en la UE y de otro prevenir los riesgos derivados de su uso

\subsection{Estructura}

En general, la "Estructura para el Libro Blanco sobre Al un enfoque europeo" debe ser reputado como un documento orientativo y fundacional que busca proporcionar una visión general de la disposición reguladora de la tierra para la IA en la UE y propone varias opciones para las implementaciones de futuras normativas. Establece el marco político existente para la IA a nivel de la UE y más allá; describe por qué la IA necesita ser promovida en toda Europa; propone un mejor acceso a los datos a través de las tecnologías más disruptivas (tales como la edge computing); y profundiza en los elementos clave del futuro marco legislativo europeo integral para la IA; cerrando con cinco opciones de gobernanza.

Este documento persigue, desde un enfoque muy europeo, convertir a la Unión Europea en líder global de la data-economy y sus aplicaciones, en beneficio de ciudadanos, negocios y sociedad en general, sobre la base de dos pilares o ejes fundamentales para lograr este objetivo, claramente marcados, el ecosistema de excelencia y el ecosistema de confianza. 


\subsection{Ecosistema de la excelencia}

Dentro de este objetivo se busca optimizar las acciones sobre la cadena de valor del dato, tanto desde una perspectiva pública como privada. En esta parte se contemplan seis acciones relativas a los siguientes aspectos:

Se plantea la necesidad de coordinación con los estados miembros, elaborando un plan coordinado cuya publicación final apunta a finales de 2020. Asimismo, se propugna la necesidad de implemente un foco en I+D, lo que conlleva la necesaria creación de centros de excelencia europeos centralizados. El documento recalca la imperiosa necesidad de incrementar las capacidades existentes tanto para la digitalización de la sociedad como relativos a la formación de profesionales intracomunitarios y a la atracción de talento del exterior

Otro de los aspectos a resaltar, visto el extraordinario peso que tiene sobre la economía de la UE, consiste en la búsqueda el foco en pymes, facilitando la digitalización de las pequeñas y medianas empresas a través de hubs de innovación digital en cada país miembro. Vista la escasa participación del sector privado en este campo, comparando la UE con China o EE. UU, se busca profundizar en los canales de cooperación entre el sector público y el privado. Ya en el campo de adopción de la IA por parte del sector público, se presta especial atención especial al sector de la salud, de las administraciones rurales y las operadoras de servicios públicos.

Del mismo modo, el documento analiza la problemática de la seguridad en el acceso a los datos e infraestructuras, así como las bases para la cooperación internacional, fundamentalmente en el ámbito de la OCDE.

\subsection{Ecosistema de confianza}

Cual rostro de J ano, esta es la otra cara de la moneda; este apartado cobija el enfoque humanista que tiende a controlar los potenciales excesos y abusos que comporta el uso del enorme poder que ostenta la IA. En este ecosistema conviene recordar los ya mencionados siete requerimientos apuntados en las Pautas éticas para una IA confiable. 
A su vez los 7 requerimientos pueden ser subsumidos en tres pautas: en primer lugar, la legalidad o el respeto a todas las normativas y regulaciones aplicables; en segundo lugar, la ética o el respeto a los principios y valores éticos: la robustez técnica, tanto desde una perspectiva técnica teniendo en cuenta su entorno social.

El requisito 1 se intitula "Humanismo y supervisión" que implica que los sistemas de IA deberían dar el poder de la decisión final a los seres humanos, permitiéndoles tomar decisiones informadas y fomentar sus derechos fundamentales. Al mismo tiempo, deben garantizarse mecanismos de supervisión adecuados, que pueden lograrse a través de algún tipo de presencia humana en la toma de la decisión.

El 2.o requisito viene referido a la robustez técnica y a la seguridad. Implica que los sistemas de IA deben ser resistentes y seguros, garantizando un plan alternativo en caso de que algo salga mal, además de ser precisos, confiables y reproducibles. Se considera que esa es la única forma de garantizar que también se puedan minimizar y prevenir daños no intencionales.

El tercer requerimiento viene referido a la privacidad y gobernanza. Además de garantizar el pleno respeto de la privacidad y la protección de datos, también deben garantizarse mecanismos adecuados para la buena gestión de los datos, teniendo en cuenta su calidad e integridad, y garantizando el acceso legítimo a los mismos.

El 4. requerimiento viene referido a la transparencia que alude a que los datos, el sistema y los modelos de negocio de IA deben ser transparentes. Los mecanismos de trazabilidad pueden ayudar a lograr este objetivo. Además, los sistemas de IA y sus decisiones deben explicarse de un modo accesible que se adapte a las características de las partes implicadas. Los seres humanos deben ser conscientes de que están interactuando con un sistema de IA y, en consecuencia, deben ser convenientemente informados sobre las capacidades y limitaciones del sistema. Este tema se encuentra interrelacionado con el denominado right to legiblity ${ }^{63}$

63 Vid. Goodman, Bryce / Flaxman, Seth, "European Union regulations on algorithmic decision-making and a "right to explanation"”, Al Magazine, Vol.38 Núm.3, 28 de junio de 2016. 
El 5.. requerimiento se denomina "diversidad, no discriminación y equidad". Se deben evitar los agravios comparativos, ya que podría tener múltiples implicaciones negativas, desde la marginación de los grupos vulnerables hasta la exacerbación de los prejuicios y la discriminación. Al fomentar la diversidad, los sistemas de IA deben resultar accesibles para todos, independientemente de cualquier discapacidad, e involucrar a las partes interesadas relevantes a lo largo de todo su círculo de vida.

El sexto requerimiento viene referido al "bienestar social y medioambiental". Los sistemas de IA deberían beneficiar a todos los seres humanos, incluidas las generaciones futuras. Por lo tanto, debe garantizarse que sean sostenibles y respetuosos con el medio ambiente. Además, deben tener en cuenta el medio ambiente, incluidos otros seres vivos, y su impacto social debe considerarse cuidadosamente. Dada su parte, la materia medioambiental es objeto de un estudio más extenso.

El último requisito es la responsabilidad. El documento propone que deben implantarse mecanismos para garantizar la responsabilidad y la rendición de cuentas para los sistemas de IA y sus resultados. La posibilidad de ser auditados permite la evaluación de algoritmos, datos y procesos de diseño ${ }^{64}$. Se considera que esta posibilidad de revisión desempeña un papel clave, especialmente con relación a las aplicaciones consideradas críticas.

\section{$\underline{7.4 \text { Objetivos }}$}

El documento de estrategia digital publicado por la comisión establece cómo pretende posicionar estratégicamente a Europa como un "marcador de tendencias" con respecto a los datos, específicamente a través de tres objetivos clave relacionados con las tecnologías digitales en los próximos cinco años:

Un primer pilar sería la tecnología que funciona para las personas. Los objetivos de este objetivo son la tecnología que marca la diferencia en la vida cotidiana de las personas y la

64 Vid. Burrell, Jenna, “How the machine 'thinks': Understanding opacity in machine learning algorithms", Big Data \& Society, Vol. 3, Núm. 1, 6 de enero de 2016, pp.1-12. La autora refiere que la opacidad de la IA viene relacionada con los sistemas de algoritmos inherentes a la IA. 
racionalización de la colaboración y la interoperabilidad con respecto a los datos. Un segundo apartado es denominado la economía justa y competitiva. Este objetivo se centra en crear un mercado único sin fricciones con competencia en igualdad de condiciones y el respeto de los derechos de los consumidores.

Finalmente, la IA debe emerger en el marco de una sociedad abierta, democrática y sostenible. El objetivo aquí es crear un entorno confiable con ciudadanos empoderados, mejorar los valores democráticos y contribuir a una economía sostenible.

Crear un "marco legislativo operativo para la gobernanza de los espacios comunes de datos europeos" que llegará en el cuarto trimestre de 2020. La idea es que se pueda usar para tomar decisiones en torno a qué datos pueden usarse según en qué situaciones, facilitar la utilización transfronteriza y dar prioridad a los requisitos de interoperabilidad.

Se busca democratizar los datos y para ello se postula la necesidad de implementar una "ley de aplicación de los conjuntos de datos de alto valor" en el primer trimestre de 2021 basada en la Directiva de Datos Abiertos. Su objetivo será abrir los conjuntos de datos de referencia del sector público; es decir, ponerlos a disposición de los usuarios de forma gratuita en toda la UE, convirtiéndolos a un formato legible por las máquinas a través de APIs estandarizadas.

En línea con lo anterior, la UE estudiará la necesidad de adoptar medidas legislativas sobre las cuestiones que afectan las relaciones entre los agentes de la economía de la información a fin de ofrecer incentivos para el intercambio horizontal de datos entre los sectores. Todo esto se desarrollará en la Data Act de 2021.

\section{$\underline{7.5 \text { Riesgos }}$}

A continuación, sobre la base de los pilares descritos en dicha guía, el documento repasa la problemática fundamental asociada a la necesidad de una inteligencia artificial que aporte confianza a la sociedad, cubriendo dos tipos de riesgos. 
Para los derechos fundamentales, principalmente sobre datos personales, privacidad y discriminación. Peligros para la seguridad de las personas y a la asunción de responsabilidades derivadas del uso de la IA. Para ello se contempla la necesidad de abordar cambios legislativos que conduzcan a la Unión hacia un nuevo marco regulatorio de la economía del dato, especialmente en lo que se denomina la Inteligencia Artificial de Alto Riesgo (HRAI, por sus siglas en inglés), definida como aquellas aplicaciones en las que se conjugan dos criterios:

a. Riesgo en función de la naturaleza del ámbito. Aquí se incluyen sectores como la salud, energía, transporte, algunos servicios públicos... en una lista que ha de completarse en el Plan definitivo.

b. Riesgo en función del uso final, lo que significa que no todos los usos que se hagan de la IA en los sectores considerados de alto riesgo serían incluidos en la categoría HRAI. Pensemos por ejemplo en la gestión de las listas de espera de un hospital, como un uso de bajo riesgo en un sector de alto riesgo.

Una vez inventariados los usos de alto riesgo, la cuestión radica en imponer una serie de requerimientos asociados a los diferentes aspectos del proceso:

Sets de datos de entrenamiento, donde las preocupaciones principales estriban en la seguridad, la privacidad y los sesgos discriminatorios.

Documentación y archivo de los procesos, en cuanto a criterios de selección de datos, algoritmos empleados, etc.

Otros aspectos como la gobernanza de las políticas y acciones acordadas, la aplicabilidad a terceros extracomunitarios, el compliance y la posibilidad de adscripción voluntaria en el caso de usos de bajo riesgo, son también comentados brevemente en el documento.

En definitiva, la Comisión Europea, consciente del retraso de la Unión frente a Estados Unidos y China, está sentando las bases para la fundación de un modo europeo de entender la 
data-economy, basado en la aportación de valor a la sociedad sobre la base del respeto a los derechos humanos, en una suerte de humanismo tecnológico.

\subsection{Importancia de las implicaciones medioambientales}

El documento anhela convertir a Europa en un líder en innovación en la economía de datos, así como sus aplicaciones ${ }^{65}$. Para ello parte sus fortalezas tecnológicas e industriales con una alta calidad infraestructura digital y un marco regulatorio basado en su enraizado respeto a los fundamentales.

Para el documento, la revolución digital no puede ser desligada de los abiertos retos medioambientales. Se analiza que el impacto de los sistemas de IA debe ser contemplado no solo bajo una perspectiva individual (uti singulis), sino también desde la óptica del impacto que va a tener sobre el conjunto social (uti socius). Se entiende que el uso de sistemas de IA puede ejercer un rol trascendental en el logro de los Objetivos de Desarrollo Sostenible, y en el apoyo al proceso democrático y social de los derechos. Con sus recientes propuestas sobre el Acuerdo Medioambiental Europeo ${ }^{66}$. Dado que Europa ha tomado un papel activo en relación con los desafíos climáticos y medioambientales, se considera que las tecnologías digitales como la IA deben ser instrumentalizadas como una crucial herramienta para alcanzar los objetivos del acuerdo medioambiental. Dada la creciente importancia de la IA para el medio ambiente.

En relación con la economía circular el impacto de los sistemas de IA debe considerarse debidamente a lo largo de su ciclo de vida y en toda cadena de suministro, en cuanto al uso de recursos para la formación de algoritmos y el almacenamiento de datos.

Otro de los elementos clave de un futuro marco regulatorio para la IA en Europa que creará un entorno único. 'Ecosistema de confianza'. Para hacerlo, debe garantizar el cumplimiento de las normas de la UE, incluidas las normas proteger los derechos

65 COM (2020) 66 parte final.

66 COM (2019) 640 parte final. 
fundamentales y los derechos de los consumidores, en particular para los sistemas de IA operados en la UE que presenta un alto riesgo ${ }^{67}$.

Se plantea como un objetivo político en sí mismo erigir un ecosistema de confianza, y debería dar a los ciudadanos la confianza para aceptar aplicaciones de IA y dar a las empresas la seguridad jurídica de innovar utilizando IA. Partiendo del texto del documento, la comisión trata de conjugar las innovaciones tecnológicas con el respeto a una perspectiva humanista centrado en el respeto al ser humano $\mathrm{Al}^{68}$ y también tendrá en cuenta la aportación obtenida durante la fase piloto la directrices éticas preparadas por el grupo de expertos de alto nivel sobre IA.

\section{Conclusiones}

Irrefutablemente la IA es uno de los grandes dilemas con los que se va a enfrentar la humanidad de cara al futuro, su potencial es tan prodigioso que puede comportar la extinción de la especie humana, pero también abre la puerta de colocar al ser humano a una escala universal. Europa se encuentra retrasada en materia de inversiones en relación con la IA, comparándola con otras megapotencias mundiales tales como EE. UU., China o India.

No obstante, como el propio documento analizado apunta, existen determinadas variables que convenientemente utilizadas pueden generar sinergias que la coloquen en cabeza en el mundo de la IA. Como todo artilugio artificial, la IA comporta un componente mecánico o deshumanizador. La UE, a diferencia de otras potencias, busca acomodar las expectativas técnicas y humanas en aras evitar convertir un espacio de convivencia democrático en un mundo orwelliano. El pésimo ejemplo de mal uso de la IA lo encontramos en el caso del crédito social chino donde el ciudadano opera como una mera pieza de engranaje de un sistema.

$67 \mathrm{Si}$ bien se considera necesario implementar más medidas para prevenir y contrarrestar el mal uso de la IA con fines delictivos, el documento aclara que dicho objetivo está fuera del alcance del analizado Libro Blanco.

$68 \operatorname{COM}(2019) 168$. 
Vivimos en la sociedad del riesgo ${ }^{69}$. Las sociedades avanzadas generan numerosos beneficios, pero a la par potenciales riesgos autodestructivos. El derecho (y la UE no deja de ser una comunidad jurídica pues es lo único que le da vida) ocupa un rol importante de regulación y gestión de esos innumerables riesgos (atómicos, bilógicos, medioambientales, sanitarios, etc.) riesgos que se explican y encuentran su sentido en el modelo de sociedad telematizada que los cobija.

Paralelamente, el Libro Blanco de la UE para la I A descansa en este comentado esquema bipolar: de un lado el ecosistema de la excelencia, orientado a maximizar los ineludibles beneficios de la IA. La UE cree que es necesario aumentar significativamente la inversión en investigación en IA, puesto que solo en 2016 se invirtieron 3,2 billones de euros en Europa, mientras que en Estados Unidos y Asia la inversión fue de 12,1 y 6,5 billones, respectivamente. Asimismo, crear centros de excelencia y de pruebas que puedan combinar inversiones europeas, nacionales y privadas, posiblemente incluyendo un nuevo instrumento jurídico. La comisión ha propuesto una cantidad ambiciosa y específica para apoyar a los centros de pruebas de referencia mundial en Europa en el marco del Programa Europa Digital y se complementará, cuando proceda, por las actividades de investigación e innovación de Horizonte Europa como parte del Marco Financiero Plurianual para 2021-2027.

Otro necesario incentivo radica en establecer y apoyar, a través del pilar de conocimientos avanzados del Programa Europa Digital, redes de las principales universidades e institutos de enseñanza superior para atraer a los mejores profesores y científicos y ofrecer programas de maestría en IA de primer nivel mundial.

La otra cara de la moneda es el ecosistema de la confianza. orientado a conjurar los peligros para una sociedad democrática avanzada que comporta un mundo digitalizado. Buscando un equilibrio en la balanza, UE postula que para aprovechar las oportunidades y sobrepasar los retos que supone la implantación de la IA, se debe actuar basándose en "valores europeos"

69 Vid. BECK, Ulrich, La sociedad del riesgo: hacia una nueva modernidad, Paidós Ibérica, Barcelona, 2006. 
(entendido fundamentalmente como un respeto a los derechos fundamentales y al principio democrático) y promoviendo el desarrollo y despliegue de la IA.

Uno de los aspectos positivos del Libro Blanco es la democratización de la información. Este propósito se buscará alcanzar con la "Ley de aplicación de los conjuntos de datos de alto valor" que se aprobará en el primer trimestre de 2021 basada en la Directiva de Datos Abiertos. Su objetivo es abrir los conjuntos de datos de referencia del sector público; es decir, ponerlos a disposición de los usuarios de forma gratuita en toda la UE, convirtiéndolos a un formato legible por las máquinas a través de APIs estandarizadas.

De otro lado, el Libro Blanco sobre IA, como todo libro blanco, busca ofrecer información sobre cómo se aproxima una organización a cierto problema, en este caso, la regulación de la IA. Desde la UE se muestran a favor de un "enfoque orientado a la regulación y la inversión" con el doble objetivo de promover la adopción de la IA y de "abordar los riesgos asociados a determinados usos de esta nueva tecnología". Eso sí, siempre desde el punto de vista de un mercado único que evite la "fragmentación" y permita conseguir la suficiente escala.

Finalmente, el Libro blanco debe ser entendido como un inicio, pero no es definitivo ni mucho menos. hasta el 19 de mayo de 2020, estuvo abierto un sistema de comentarios y consultas para añadir mejoras, comentarios, etc. Además, esta propuesta depende en gran medida de la futura redacción de la Data Act de 2021.

\section{Bibliografía}

Beck, Ulrich, La sociedad del riesgo: hacia una nueva modernidad, Paidós Ibérica, Barcelona, 2006. Burrell, Jenna, “How the machine 'thinks': Understanding opacity in machine learning algorithms", Big Data \& Society, Vol. 3, Núm. 1, 6 de enero de 2016.

Chen, Hsinchun/ Zeng, Daniel/ Buckeridge, David L./ IZADI, Masoumeh/ VERMA, Aman/ OKHMATOVSKAIA, Anya/ HU, Xiaohua/ SHEN, Xiajiong/ CAO, Zhidong/ WANG, Fei-Yue/ ZHENG, Xiaolong/ WANG, Quanyi, "Al for Global Disease Surveillance", IEEE Intelligent Systems, Vol. 24, Núm.6., noviembre/ diciembre, 2009 Clutterbuck, Richard, Terrorism in an unstable world, Londres/ Nueva York, 1994.

Damasio, Antonio, The Feeling of What Happens: Body, Emotion and the Making of Consciousness, Harcourt Brace, Nueva York, 2000.

Deleuze, Gilles, Différence et répétition, PUF, Paris, 1997. 
Dennet, Daniel C., From Bacteria to Bach and Back: The Evolution of Minds, Penguin Random House Londres, 2018.

Fatemeh, Zahedi, "An Introduction to Neural Networks and a Comparison with Artificial Intelligence and Expert Systems", Interfaces, Vol. 21, Núm. 2, marzo/abril, 1991.

Ferrucci, David A./ LEVAS, Anthony/ Bagchi, Sugato/ Gondek, David / Mueller, Erik T., "Watson: beyond jeopardy!", Artificial Intelligence, Núm. 199, 2013.

Foucault, Michel, Panóptico: El Ojo del poder, La Piqueta, Madrid, 1979. Microfísica del poder, La Piqueta, Madrid, 1979.

Gardner, Esther P. / Martin, John H., "Codificación de la información sensorial" en Principios de neurociencia (Kandel, Erik R., et al coords), Mc Graw Hill, 1aㅡ Ed., Madrid, 2001.

Goodman, Bryce / Flaxman, Seth, "European Union regulations on algorithmic decision-making and a "right to explanation"", Al Magazine, Vol.38 Núm.3, 28 de junio de 2016.

Gubern Garriga, Román, El Simio informatizado, Ed., Fundesco, Madrid, 1987.

Kamley, Sachin / Jaloree, Shailesh /Thakur, Ramjeevan Singh, "Performance Forecasting of Share Market using Machine Learning Techniques: A Review", International Journal of Electrical and Computer Engineering Vol. 6, Núm. 6, diciembre de 2016.

Krauthammer, Charles, "Be afraid", Washington Examiner, 26 de mayo de 1997, recuperado de https://www.washingtonexaminer.com/weekly-standard/beafraid-9802 (última visita 12 de marzo de 2020).

Lomas, Nastasha,"Study: Russian Twiter bots $45 \mathrm{k}$ bots tweets close to vote", 15 de noviembre de 2017, recuperado de (https://techcrunch.com/2017/11/15/ study-russian-twitter-bots-sent-45k-brexit-tweets-close-to-vote/, última visita 3 de marzo de 2020).

Majó Roca, Joan, Chip, cables y poder: la clase dominante en el siglo XXI, Planeta, Barcelona, 1997.

Maslow, Abraham, Toward a psychology of being, 2ª . Ed., Van Nostrand, Nueva York, 1968.

- The farther reaches of human nature, Viking Press, Nueva York, 1971.

Masuda, Yoneji. La sociedad informatizada: como sociedad post-industrial, Tecnos, Madrid, 1984.

Mora, Francisco, El reloj de la sabiduría, Tiempos y espacios en el cerebro humano, Alianza Ed., Madrid, 2002.

Moreno, Luis/ Jiménez, Ramón, Democracias Robotizadas. Escenarios futuros en Estados Unidos y la Unión Europea, Eds. Catarata, Madrid, 2018.

Mostrous, Alexei/ Bridge, Mark/ Gibbons, Katie, "Russia used Twitter bots and trolls 'to disrupt' Brexit vote", The Sunday Times, 15 de noviembre de 2017, recuperado de (https://www.thetimes.co.uk/edition/news/russia-used-web-posts-todisrupt-brexit-vote-h9nv5zg6c, última visita 3 de marzo de 2020).

Pinker, Steven, La tabla rasa: La negación de la naturaleza humana, (trad. Filella Escolà, Roc), Paidós, Barcelona, 2003.

- Cómo funciona la mente, (trad. Ferran Meler-Orti), Destino, Barcelona, 2008.

Rodrigo Alsina, Miquel, "El conocimiento del sistema penal: alarma social y medios de comunicación", Cuadernos de Derecho Judicial, Núm. IV, 1999.

Searle, John R., "Minds, brains, and programs", Behavioral and Brain Science, Vol. 3, Núm. 3, 1980.

Silver, David/ Huang, Aja/ Maddison, Cristopher J../ Guez, Arthur/ Sifre, Laurent/ Ven Den Driessche, George/ Schrittwieser, Julian/ Antonoglou, I oannis/ Panneershelvam, Veda/ Lanctot, Marc/ Dieleman, Sander/ Grewe, Dominik / Nham, J ohn / Kalchbrenner, Nal / Sutskever, Ilya/ Lillicrap, Timothy/ Leach, Maddy / Kavukcuoglu, Koray/ Graepel, Thore / Hassabis, Demis, "Mastering the game of go with deep neural networks and tree search", Nature, Vol. 529, Núm. 7.587, 2016. 
Silver, David/ Schrittwieser, Julian/ Simonyan, Karen/ Antonoglou, Ioannis/ Huang, Aja;/ Guez, Arthur/ Hubert, Thomas/ Baker, Lucas/ Lai, Matthew/ Bolton, Adrian/ Chen, Yutian/ Lillicrap, Timothy;/ Fan, Hui/ Sifre, Laurent/ Driessche, George van den/ Graepel, Thore/ Hassabis, Demis, "Mastering the game of Go without human knowledge", Nature, Vol. 550, Núm. 7676, 19 de octubre de 2017.

Taigman, Yaniv/ Yang, Ming / Ranzato, Marc Aurelio/ Wolf, Lior, "DeepFace: Closing the Gap to Human-Level Performance in Face Verification," 2014 IEEE Conference on Computer Vision and Pattern Recognition, Columbus, OH, junio de 2014.

Terceiro Lomba, J osé Buenaventura, Sociedad digital: del homo sapiens al homo digitalis, Alianza Editorial, Madrid, 1996.

Weizenbaum, Joseph, Computer Power and Human Reasoning: From Judgment to Calculation, San Francisco, W. H. Freeman and Company, 1976.

Wilson, Hilary, Understanding Hieroglyphs: A Complete Introductory Guide. Michael O'mara Books Ltd, Londres, 1965.

Von MISES, Ludwig, La acción humana, (trad. Reig Albiol, Joaquín), 6a Ed. Madrid, 2001.

Zamiátin, Evgueni Ivanovich, Nostros, (trad. Hernández-Ranera Sergio / Moreno, Fernando Ángel), Ed. Akal, Madrid, 2008. 